\title{
Efecto de la suplementación con $17 \beta$ - estradiol sobre el aprendizaje y memoria en ratas ovariectomizadas sometidas a una tarea de tipo espacial
}

Díaz-Martínez RM*, Padilla-Gómez E**, Díaz-Miranda SY***

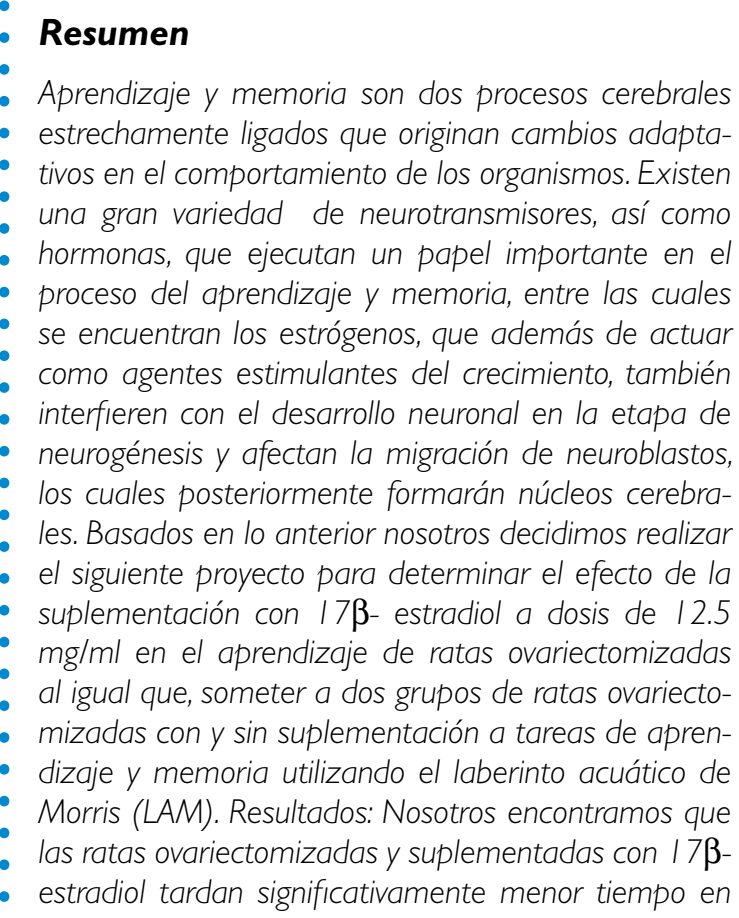

recorrer el LAM que las ratas ovariectomizadas y no suplementadas en el día uno del estudio $(p<0.05)$.

Al medir memoria a las 24 horas posterior a un día de entrenamiento y después de 10 ensayos no se observa ninguna diferencia estadística, sin embargo a las 24 horas después de 2 días de entrenamiento se aprecia una diferencia significativa con $p<0.05$. Discusión: Los resultados que se obtuvieron nos permiten demostrar que los estrógenos aumentan la capacidad plástica de áreas específicas para el aprendizaje y la memoria, como el hipocampo. Sin embargo la diferencia que se encuentra entre las ratas suplementadas y las ovariectomizadas, no es lo bastante significativa como para afirmar que el efecto de los estrógenos aumenta la capacidad de aprendizaje, sino más bien, lo que se observa con la suplementación es la reversión de los efectos adversos que produce la deficiencia de estrógenos en el sistema nervioso, lo cual traduce un efecto neuroprotector de los estrógenos. LUXMÉDICA 2010;5(14): 43-53

Palabres clave: Estrógenos, 17ß-estradiol, hipocampo, plasticidad neuronal.

* Estudiante del noveno semestre de medicina UAA.

* Estudiante de doctorado. Instituto de Neurobiología UNAM, Juriquilla, Querétaro.

*** Investigador titular C del departamento de Neurobiología del desarrollo y Neurofisiología del Instituto de Neurobiología UNAM, Juriquilla, Querétaro 


\section{Introducción}

Aprendizaje y memoria son dos procesos cerebrales estrechamente ligados que originan cambios adaptativos en el comportamiento de los organismos. El estudio de la memoria tiene diferentes vertientes, en disciplinas muy diversas, por tanto no se explica como un fenómeno fisiológico aislado de las funciones cognitivas y el entorno social, cultural y afectivo. Es interesante correlacionar las cualidades funcionales del cerebro humano con los diferentes factores endógenos y exógenos que afectan principalmente a la memoria y al aprendizaje. Así, existe una correlación entre la morfo-patología dependiente de la edad y los trastornos existentes por cambios hormonales y del envejecimiento. Las nociones actuales del conocimiento estructural, funcional y molecular de nuestro cerebro nos han permitido conocer el efecto que ciertas hormonas tienen en él, como moduladores endógenos de procesos neurobiológicos ${ }^{1,2}$.

En estructuras pertenecientes al sistema límbico, se han encontrado cambios ocurridos por la disminución de las hormonas en la mujer durante los periodos de climaterio y menopausia. Además, las alteraciones celulares se relacionan con la degeneración neuronal por edad y el Alzheimer; que tiene una prevalencia mayor en mujeres en edades posmenopáusicas. En algunos estudios se ha encontrado que el hipocampo, como parte del sistema límbico, es vulnerable al envejecimiento, al estrés y a la isquemia que producen daño y pérdida neuronal en las células de proyección o piramidales del campo CA $1 .{ }^{3}$ Sin embargo también se ha demostrado la probable protección de las células de CA1 del hipocampo, con tratamiento preventivo con $\beta$ estradiol en la isquemia ${ }^{4}$. Siendo esto un perfil demostrativo de las influencias endógenas sobre la estructura neuronal donde se vinculan los esteroides sexuales.

Además de su participación en la formación de la memoria, el hipocampo participa en el control del sistema endócrino y en la regulación del sistema inmunológico ${ }^{5}$. En la mujer la regulación endócrina juega un papel importante tanto en su comportamiento sexual como psicológico. En el ovario se secretan dos clases de hormonas: estrógenos y progestinas; siendo las más importantes el estradiol y la progesterona respectivamente. Además de sus funciones biológicas a nivel de las características sexuales de la mujer, favorecen el crecimiento óseo estimulando la actividad de los osteoblastos y aumentado ligeramente las proteínas corporales y la intensidad del metabolismo y a nivel del sistema nervioso central se les han propuesto diferentes funciones ${ }^{6}$. Además estas hormonas participan en procesos cognitivos esenciales como el aprendizaje y memoria, al igual tiene significancia en la regulación del estrés y la depresión, aunado a esto, este tipo de esteroides apoyan a una de las funciones vitales para el sistema nervioso como es la neuroprotección. 


\section{Aprendizaje y memoria}

El aprendizaje es un proceso por el que los organismos modifican su conducta para adaptarse a las condiciones cambiantes e impredecibles del medio que los rodea. Junto a las fuerzas selectivas de la evolución, el aprendizaje constituye el modo principal de adaptación de los seres vivos. Cuanto más cambiante es el entorno, más plástica debe ser la conducta, por lo que los organismos que viven en medios diferentes presentan también grados diferentes de plasticidad conductual. Esta plasticidad es reflejo a su vez de la que caracteriza a las neuronas y al sistema nervioso de los organismos. Cuanta más plasticidad tiene su sistema nervioso más posibilidades de aprendizaje tiene un animal. Por tanto, el aprendizaje puede considerarse como un cambio en el sistema nervioso que resulta de la experiencia y que origina cambios duraderos en la conducta de los organis$\operatorname{mos}^{7}$.

En la última década del siglo XIX, Santiago Ramón y Cajal propuso una teoría del almacenamiento de la información, que postula que la información se almacena en el cerebro mediante cambios anatómicos entre las conexiones de las neuronas. Así mismo, Sherrington propuso el término plasticidad sináptica para describir los cambios en las propiedades funcionales de una sinapsis como resultado de su actividad; este término se refiere a la posibilidad de inducir cambios que pueden o no ser reversibles ${ }^{8}$.

\section{El procesamiento de la información, la memoria y el aprendizaje}

La información debe ser codificada antes de ser almacenada en la memoria a largo plazo. Esta codificación de la información pertinente es realizada por el hipocampo durante el sueño profundo y almacenada en diferentes lugares del cerebro. La información se vuelve a reunir para formar el recuerdo. Según el modelo la informa- ción es procesada a través de las memorias temporales, codificada por el hipocampo y almacenada en diferentes lugares del cerebro en lo que se denomina memoria de largo plazo. Tendemos a recordar lo que tiene sentido y significado para nosotros y a descartar aquello que no comprendemos y a lo que no le encontramos utilidad. Las emociones aumentan la intensidad de nuestras vivencias generando un recuerdo más fuerte y permanente ${ }^{9}$.

Si tomamos la definición de Sousa sobre el aprendizaje y la memoria que señala que el aprendizaje es "el proceso mediante el cual adquirimos nuevos conocimientos y habilidades" y la memoria "el proceso mediante el cual retenemos el conocimiento y las habilidades para utilizarlas en el futuro" podemos concluir que el aprendizaje solo se logra si logramos retener estos conocimientos y habilidades para utilizarlos en el futuro, actividad que realiza nuestra memoria. La memoria a largo plazo se lleva a cabo en dos etapas. La primera durante el periodo de vigilia, codifica rápidamente la información en la región CA3 del hipocampo como representación episódica de mediana duración y facilitación de las células piramidales. La segunda ocurre durante el reposo vigil y el sueño de ondas lentas teta y activación del hipocampo con ondas rápidas y espigas, fortaleciendo las sinapsis excitatorias de CA3 y CA1. Se activan las conexiones del hipocampo con la corteza entorrinal para formar memorias semánticas y episódicas a largo plazo en el neocórtex, cambios modulados por acetilcolina. Durante el reposo y el sueño disminuye la acetilcolina del hipocampo (regiones CA1 y CA3) y corteza prefrontal liberando las sinapsis glutamatérgicas de la inhibición colinérgica permitiendo mejor conexión del hipocampo con la corteza entorrinal y asociativa. Para la consolidación es esencial la integridad de la modulación colinérgica en los ciclos sueño-vigilia. La serotonina facilita las sinapsis, con un receptor metabo- 
trópico asociado a proteína-G, activando la adenilciclasa y aumentando AMP cíclico en las neuronas sensoriales ${ }^{10}$

\section{Hipocampo}

El hipocampo se sitúa en el lóbulo temporal. Forma una parte del sistema límbico y participa en la memoria y la orientación espacial. Aunque se carece de consenso en lo referente a los términos que describen el hipocampo y la corteza cerebral adyacente, el término formación hipocampal se aplica al giro dentado, los campos CA1CA4 del asta de Amón, y el subículo. Las regiones CA1, CA2 y CA3 componen el hipocampo propiamente dicho. La información atraviesa de la circunvolución del dentado a CA3 a CA1 al subículo. La vía perforante, la cual trae información sobre todo de la corteza entorrinal generalmente se considera la fuente principal de entra$\mathrm{da}$ al hipocampo. Los caminos principales de la salida del hipocampo son el cíngulo y las fimbrias del fórnix, que se presentan en la región CA1 y el subículo. La trayectoria de entrada de la vía perforante a la capa II de la corteza entorrinal se retransmite a la región CA3. Alternadamente, CA1 proyecta al subiculum así como enviar la información a lo largo de las trayectorias ya mencionadas de la salida del hipocampo. El subiculum es la etapa final en el camino, combinando la información de la proyección CA1 y de la capa III de la EC también para enviar la información a lo largo de los caminos de la salida del hipocampo ${ }^{11}$.

\section{Funciones mnemotécnicas}

No se ha llegado a un consenso sobre el papel exacto del hipocampo, pero generalmente se está de acuerdo que tiene un papel esencial en la formación de nuevos recuerdos asociados a la experiencia o memoria autobiográfica. Está relacionado fun- cionalmente con la memoria, en concreto con la retención a largo plazo de la información espacial ${ }^{12}$. Existe cierta evidencia que implica que el hipocampo almacena y procesa la información espacial. Los estudios en ratas han demostrado que las neuronas en el hipocampo tienen regiones especiales de activación. Estas células se denominan célula de lugar. Algunas células se activan cuando el animal se encuentra en una ubicación particular, sin importar la dirección del recorrido, mientras que la mayoría es parcialmente sensible a la dirección principal y a la dirección del recorrido ${ }^{13}$.

Fisiológicamente, los recuerdos se producen por variaciones de la sensibilidad de transmisión sináptica de una neurona a la siguiente como resultado de la actividad neural previa. Esta variaciones a su vez generan nuevas vías o vías facilitadas de transmisión de las señales por los circuitos neurales del cerebro. Las vías nuevas o facilitadas se denominan huellas de la memoria. Son importantes debido a que, una vez establecidas, la mente pensante puede activarlas para reproducir los recuerdos ${ }^{14}$. Los hipocampos en especial y en menor medida los núcleos dorsomediales del tálamo, resultan esenciales para decidir qué pensamientos tienen la importancia suficiente, basándose en la recompensa o en el castigo para ser recordados. El hipocampo tiene vías de comunicación bidereccionales con todos los niveles del sistema límbico, y otras principalmente indirectas con muchas porciones de la corteza cerebral. Otra característica del hipocampo es su hiperexcitabilidad habitual, probablemente porque su corteza difiere del resto del cerebro, ya que solo posee tres capas de células nerviosas en algunas partes en vez de seis capas de otras zonas 6 .

En una etapa muy temprana del desarrollo evolutivo del encéfalo, el hipocampo se 
convirtió probablemente en un mecanismo neuronal crítico para la toma de decisiones. Se ha sugerido que el hipocampo proporciona el impulso para la transferencia desde la memoria a corto plazo a la memoria a largo plazo, es decir, que éste transmite algún tipo de señal o señales que parecen repetir en la mente una y otra vez la nueva información hasta su almacenamiento permanente. Sea cual sea el mecanismo, sin los hipocampos no tiene lugar la consolidación del pensamiento verbal o simbólico a largo plazo ${ }^{6}$.

\section{Sinaptogenésis en el hipocampo}

Uno de los cambios estructurales que se han asociado al aprendizaje y la memoria es la sinaptogénesis. En el hipocampo se ha descrito que ocurre un incremento en los contactos sinápticos de las fibras musgosas (FM) después de manipulaciones experimentales ${ }^{8}$. Asimismo, se sugiere que la sinaptogénesis es un proceso que puede inducirse mediante la actividad repetida de las FM y que, por lo tanto, podría ocurrir normalmente durante períodos de actividad reiterada de las células granulares en animales intactos ${ }^{8}$. El hipocampo parece desempeñar un papel fundamental en la ejecución de tareas espaciales. En 1971, John O'Keefe y Jonathan Dostrovsky descubrieron que existen neuronas del hipocampo que se activan específicamente cuando el animal está en una localización determinada de un ambiente. Por ello, se les Ilamó células de lugar; anatómicamente, estas neuronas son células piramidales de la región CA1 y CA3. Se ha propuesto que las células de lugar forman parte de un mapa cognitivo en el hipocampo, y se ha considerado a estas células como elementos de una representación cartesiana del ambiente ${ }^{8}$.
Existen una gran variedad neurotransmisores así como hormonas que ejecutan un papel importante en el proceso del aprendizaje y memoria entre las cuales se encuentran las hormonas esteroideas como los estrógenos, de los cuales se ha demostrado su participación tanto en la sinaptogénesis, así como en la espinogénesis y neurogénesis del hipocampo.

\section{ESTRADIOL}

El estradiol ( $E_{2}$ o $17 \beta$ - estradiol) no sólo tiene un impacto crítico sobre el funcionamiento sexual y reproductivo, sino que también afecta a otros órganos. Se sintetiza a partir de la conversión de la testosterona a estradiol, al igual que otros esteroides, se obtiene a partir de colesterol. Después de la escisión de la cadena lateral del mismo y la utilización de la vía delta- 5 o la vía delta-4 se obtiene androstendiona como intermediario. Una fracción de la androstendiona se convierte a testosterona, que a su vez sufre posterior conversión a estradiol por una enzima llamada aromatasa. Alternativamente, la androstendiona es aromatizada a estrona, que posteriormente se convierten a estradiol.

El estradiol libre entra en las células e interactúa con un receptor intranuclear o de membrana. Cuando el receptor de estrógeno se ha unido, su ligando puede entrar en el núcleo de las células, y regular la transcripción de genes que conduce a la formación de ARN mensajeros y la formación de nuevas proteínas en los ribosomas. Existen dos tipos de receptores intranucleares, ER $\alpha$ y $E R \beta$, el $\beta$ a su vez tiene dos isoformas, 1 y 2 . Se han clasificado otros receptores para estrógenos de membrana, llamados $X$ y otros descubiertos recientemente llamados GPR30. 


\section{Efectos genómicos}

Las diferentes respuestas a los estrógenos en el sistema nervioso central (SNC) son debidas a la diversidad de receptores (membranales y nucleares), a la variedad de señales que éstos pueden activar, así como a la naturaleza de sus células blanco.

Los receptores $E R \alpha$ y $E R \beta$, residen en el núcleo de las células blanco, asociados con proteínas inhibitorias como las proteínas de choque térmico. Los efectos genómicos involucran la síntesis de nuevas proteínas. Los receptores nucleares de estrógenos también pueden regular la transcripción de genes mediante la modulación de factores de transcripción como el factor nuclear kappa ${ }^{\mathrm{B}}$.

\section{Efectos no genómicos}

Existen efectos extremadamente rápidos de los estrógenos sobre la excitabilidad de la membrana, la apertura de canales iónicos y señalización de neurotransmisores con receptores acoplados a proteínas G. Los estrógenos y otros esteroides son capaces de unirse a receptores específicos en la membrana plasmática, aunque la naturaleza de éstos es aún controvertida. La unión a estos receptores, puede estimular diferentes mecanismos de señalización intracelular. Recientemente, se han caracterizado receptores de estrógenos acoplados a proteínas $G$, los cuales se encuentran en el retículo endoplásmico.

\section{Efecto de los estrógenos en la plasticidad del hipocampo}

Los neuroesteroides son sintetizados en el sistema nervioso periférico y central, en astrocitos y células gliales, a partir de colesterol. El estradiol puede considerarse como neuroesteroide. Se sintetiza de novo en el cerebro o a partir de testosterona por la aromatasa P450. Las células piramidales de las regiones CA1-CA3 del hipocampo y las granulares del giro dentado contienen enzimas para la síntesis de estradiol, P450
$17 \beta$ y P450 aromatasa. La distribución cerebral de esteroides, varía en las diferentes regiones, según diferentes condiciones, por ejemplo después de situaciones de estrés. Se ha visto como los estrógenos modulan la plasticidad en CA1 a través del ciclo estral generando espinogénesis en la etapa de pro-estro, en la cual los estrógenos se encuentran en altas cantidades, también se sabe que estos cambios en las concentraciones pueden llegar a modificar la densidad sináptica ${ }^{15}$, así como la neurogénesis en el GD ${ }^{16}$

Los esteroides ováricos tienen importantes efectos sobre la función cerebral incluyendo la regulación neuroendocrina de la reproducción, el humor y la cognición, así como un efecto de neuroprotección. Las hormonas esteroides siendo lipofílicas y con bajo peso molecular cruzan la barrera hemato-encefálica y acceden al cerebro, sitio importante del metabolismo de progesterona. La concentración de esteroides en el cerebro varía durante el ciclo menstrual ${ }^{17}$.

\section{Efecto de las hormonas esteroides en el aprendizaje y la memoria}

Se ha demostrado que los estrógenos mejoran la memoria y la habilidad motora. Facilitan los procesos de maduración sináptica y están asociados con el incremento de la memoria dependiente del hipocampo. El estradiol aumenta la densidad de dendritas, estimula el sistema glutamato, los receptores NMDA y reduce la neurotransmisión GABA en neuronas del hipocampo. Los estrógenos incrementan la actividad de acetilcolintransferasa. Todas las subregiones del hipocampo son ricas en receptores GABAa. La alopregnenolona mediante la vía de receptores GABAa, produce alteraciones en el aprendizaje y memoria, inhibiendo la actividad neural de la región CA-1. En contraste, los estrógenos que antagonizan los receptores GABAa incremen$\tan$ estas funciones ${ }^{18}$. 


\section{Efectos sobre neurotrofinas}

Los agentes neurotróficos son críticos para el desarrollo, mantenimiento y restauración de la función del cerebro. Se ha propuesto que los estrógenos ejercen efectos sobre el SNC a través de la activación de neurotrofinas. Los estrógenos potencian la expresión de RNAm de agentes neurotróficos, como el factor neurotrófico derivado del cerebro (BDNF), el factor neurotrófico derivado de la glia (GDNF) y el factor de crecimiento neuronal (NGF). La habilidad de los estrógenos para activar estas neurotrofinas y sus efectos sobre el mantenimiento y restauración del SNC han sido descritos en varias regiones del cerebro, que incluyen al hipotálamo, hipocampo, mesencéfalo y la corteza. Además de actuar como agentes estimulantes del crecimiento, los estrógenos también interfieren con el desarrollo neuronal en la etapa de neurogénesis y afectan la migración de neuroblastos, los cuales posteriormente formarán núcleos cerebrales. Más tarde, en el desarrollo, cuando los circuitos neuronales ya están establecidos, los estrógenos promueven la formación de sinapsis ${ }^{19}$.

Basado en los antecedentes anteriores y tomando en cuenta que ante la presencia de estrógenos en regiones del sistema nerviosos fundamentales para el aprendizaje y la memoria como es el sistema límbico, existe cierta plasticidad neuronal (sinaptogénesis, dendrogénesis, espinogénesis, y neurogénesis), nosotros decidimos realizar el siguiente proyecto para determinar el efecto de la suplementación con $17 \beta$ - estradiol a dosis de $12.5 \mathrm{mg} / \mathrm{ml}$ en el aprendizaje de ratas ovariectomizadas al igual que, someter a dos grupos de ratas ovariectomizadas con y sin suplementación a tareas de aprendizaje y memoria utilizando el laberinto acuático de Morris.

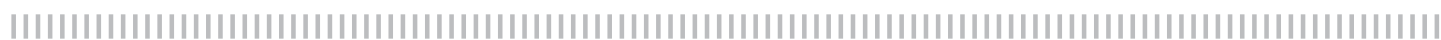

\section{Materiales y métodos}

A. Sujetos: Se usaron ratas hembras de 70 días de edad la cepa Sprague-Dawley. Se formaron dos grupos un grupo suplementado con estradiol a concentración de $12.5 \mathrm{mg} / \mathrm{ml}$ (10 ratas) y el grupo OVX (sin suplementación) (10 ratas)

B. Procedimientos: La intervención quirúrgica se redujo a una ovariectomia bilateral, bajo anestesia utilizando ketamina con xilocaína a una dosis de $0.3 \mathrm{ml}$ por vía intraperitoneal. Una vez conseguida la hipnosis se colocó la rata en decúbito supino. Por vía transperitoneal se realizó una incisión infraumbilical media de un través de dedo aproximadamente. Rechazando lateralmente el paquete intestinal para localizar las trompas de Falopio, se desprendieron los ovarios de pared posterior abdominal, se dejaron al descubierto los ova- rios, se ligaron las trompas y se seccionó la porción distal de éstas para retirar los ovarios. Inmediatamente después se colocaron las trompas e intestino en cavidad abdominal. Se suturó peritoneo y planos profundos con sutura continua con catgut, la piel se cerró con sutura continua de nylon.

Suplementación: Se inició suplementación con estradiol a dosis de $12.5 \mathrm{mg} / \mathrm{ml}$ posterior a 15 días de la ovariectomía y concordando con el día de inicio de las pruebas de aprendizaje en el laberinto acuático de Morris y los tres días consecutivos de duración de las pruebas en el LAM

\section{Prueba en laberinto acuático de Morris (LAM)}

La ejecución de la rata en el LAM fue captada por un sistema de video colocado en el techo de la habitación al centro del LAM. Las imágenes se muestran en un equipo de 
cómputo y son analizadas por el programa Smart con el que se registraron los recorridos y latencias de las ratas.

Los animales se sometieron a diez ensayos por sesión, durante dos días consecutivos en los cuales en el primer ensayo se colocó a la rata en el primer punto de salida, siempre viendo hacia la pared del tanque. Cada ensayo tuvo una duración de 60 segundos, en dicho tiempo el animal intentó buscar un punto de escape (plataforma), en dado caso en que la rata llegara a la plataforma antes de los 60 segundos, se le dejaba en ella por un tiempo de 30 segundos, y se retiraba, y en el caso en que no llegara, se le guiaba y se colocaba en la plataforma durante 30 segundos. Posteriormente se sacaba del tanque, se secaba y se dejaba descansar por 30 segundos para después dar inicio al siguiente ensayo. El mismo método se lleva a cabo de la misma manera, pero saliendo del siguiente punto de salida (2-10), sucesivamente hasta completar los diez ensayos. Este procedimiento se realizaba el día siguiente (día dos).

Con el programa Smart se midió el tiempo de latencia de llegada a la plataforma y se marcó el trayecto que realizaba la rata dentro del tanque. Para el tercer día se realizó la prueba de memoria, en la cual solo se hizo un ensayo por cada rata de dos minutos, se realizó en el LAM de igual manera, con las mismas características anteriores, solo que en esta ocasión no se colocaba la plataforma en el tanque. Como punto de inicio de la prueba se tomó el punto cardinal sur, de este punto partieron todos los animales. Usando el programa Smart se midió el tiempo que tardó cada rata en pasar por la zona en donde anteriormente estaba colocada la plataforma.

Todos los animales fueron sacrificados, después de la prueba de memoria (día tres). La mitad de cada grupo se sacrificó por decapitación a los cuales se les extrajeron ambos hipocampos en un tiempo estimado menor a 3 minutos, dichos hipocampos se congelaron a $-70{ }^{\circ} \mathrm{C}$ inmediatamente después de su resección para realizar posteriormente sobre estos el análisis de la oxidación del oxido nítrico y la expresión de proteínas relacionadas con el aprendizaje. La mitad restante se perfundió mediante la técnica de TIMM, esto para realizar posteriormente el estudio histológico y análisis estadístico de los cambios plásticos que se presentan tras la manipulación en las fibras musgosas de la región CA3 del hipocampo dorsal de la rata.

\section{| | | | | | | | | | | | | | | | | | | | | | | | | | | | | | | | | | | | | | | | | | | | | | | | | | | | | | | | | | | | | | | | | | | | | | | | | | | | | | | | | | | | | | | | | | | | | | | | | ||}

\section{Resultados}

Los resultados del análisis estadístico de la prueba de LAM fueron realizados con análisis de varianza (ANOVA) de medidas repetidas y el análisis pos hoc se hizo con una prueba exacta de Fisher. El análisis del día 1 indica diferencias estadísticamente significativas con $\mathrm{p}<0.05$ entre los grupos Ovx y Ovx-S, a partir del e5 que corresponde a los ensayos 5 y 6 (Figura 1) y en el día dos a partir del ensayo 3 que corresponden a los ensayos 15 y 16. (Figura 2). También se observan diferencias estadísticas significativas a través de los ensayos en el grupo OVX-S tanto en el día 1 como en el día 2 $(p<0.01)$. 
Aprendizaje día 1

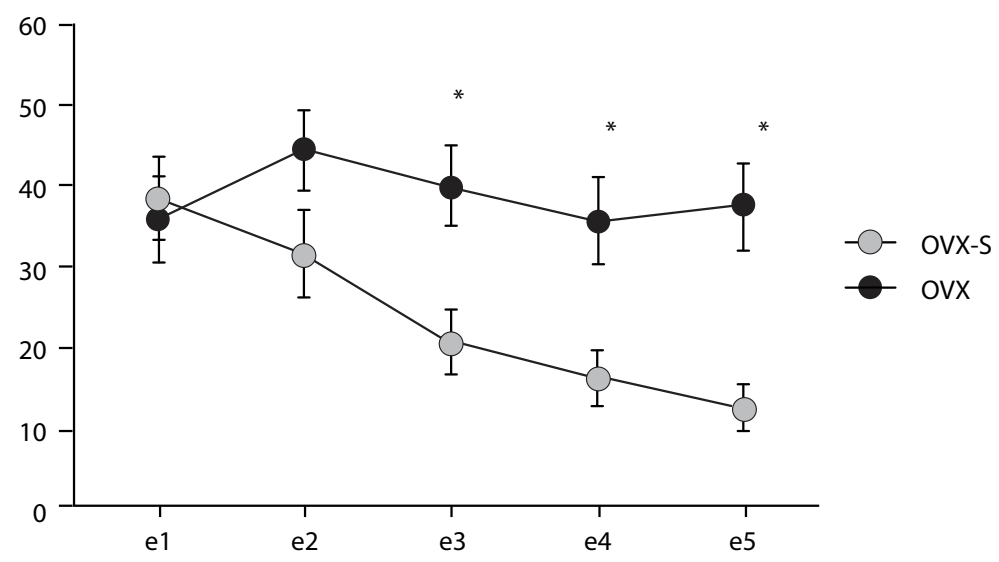

Figura 1. Ejecución en el LAM día 1. El eje de las ordenadas muestra el tiempo de llegada a la plataforma (60 segundos máximo). En el eje de las abcisas se muestran los 10 ensayos agrupados en dos, cada punto muestra el promedio del grupo en llegar a la plataforma y los asteriscos muestran diferencias estadísticamente significativas entre los dos grupos.

Aprendizaje día 2

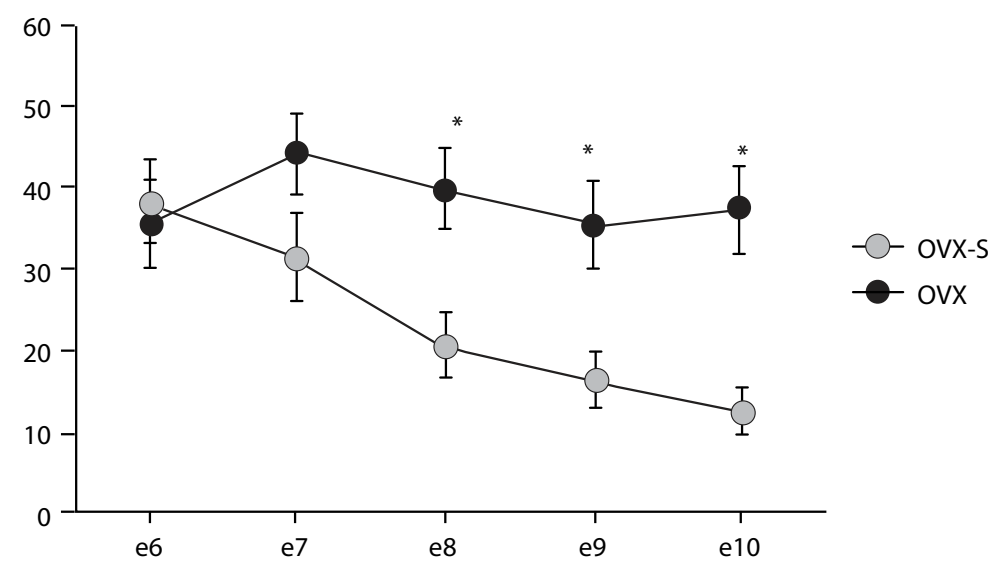

Figura 2. Ejecución en el LAM día 2. El eje de las ordenadas muestra el tiempo de llegada a la plataforma (60 segundos máximo). En el eje de las abscisas se muestran los 10 ensayos agrupados en dos, cada punto muestra el promedio del grupo en llegar a la plataforma y los asteriscos muestran diferencias estadísticamente significativas entre los dos grupos

Al medir memoria a las 24 horas posterior a un día de entrenamiento y después de 10 ensayos no se observó ninguna diferencia estadística (Figura 3), sin embargo a las 24 horas después de 2 días de entrenamiento se aprecia una diferencia significativa con $\mathrm{p}<$ 0.05 (Figura 4). 
Memoria a las 24 horas

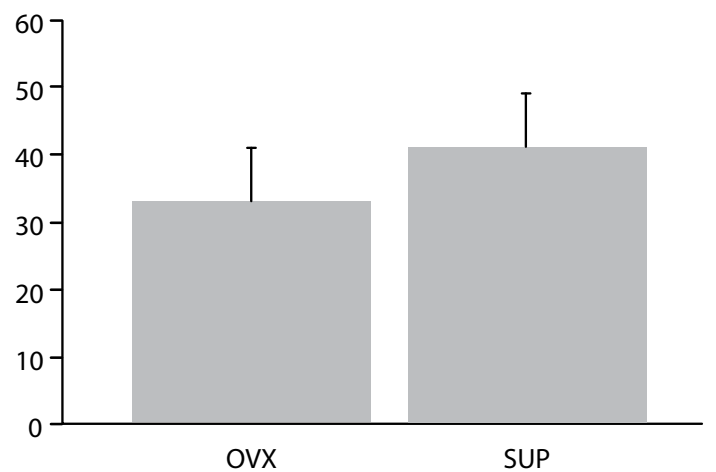

Figura 3. Memoria a las 24 horas. La gráfica representa el tiempo promedio de las 10 ratas de cada grupo, en llegar a la zona donde se encontraba la plataforma. En la cual no se muestra ninguna diferencia estadística entre ambos grupos.

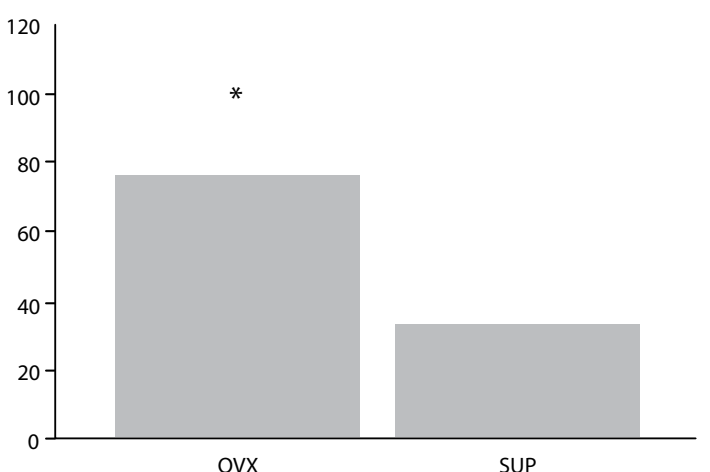

Figura 4. Memoria a las 24 horas del día 2. La gráfica representa el tiempo promedio de cada grupo de la latencia en llegar a la zona donde se encontraba la plataforma de escape, el asterisco muestra diferencias estadísticamente significativas entre los grupos.

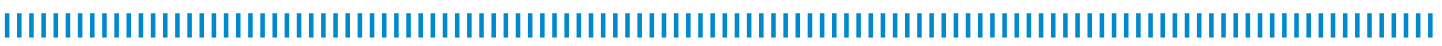

\section{Discusión}

Los estrógenos, claramente afectan el sistema cerebral involucrado en el procesamiento y almacenamiento de información. Los resultados que se obtuvieron tras la realización del proyecto, muestran concordancia con lo esperado, ya que se ha demostrado que los estrógenos aumentan la capacidad plástica de áreas específicas para el aprendizaje y la memoria, como el hipocampo. Sin embargo la diferencia que se encuentra entre las ratas suplementadas y las ovariectomizadas, no es lo bastante significativa como para afirmar que el efecto de los estrógenos aumenta la capacidad de aprendizaje, sino más bien, lo que se observa con la suplementación es la reversión de los efectos adversos que produce la deficiencia de estrógenos en el sistema nervioso, ya que como se mencionó anteriormente los estrógenos participan en la neurogénesis así como en el mantenimiento y restauración del sistema nervioso, por lo que se considerarían como neuroprotectores. Dicha reversión se ha observado en estudios histológicos y neuroquímicos realizados en cerebros de ratas hipoestrogénicas sometidas a suplementación hor- monal. Efecto que se pierde o disminuye en la postmenopausia con la disminución de los niveles hormonales sistémicos y en sistema nervioso.

Los riesgos y beneficios de la terapia de reemplazo hormonal sobre la función cognitiva en la menopausia han sido difíciles de definir. Las acciones fisiológicas de los estrógenos que podrían sustentar un efecto sobre la cognición en las mujeres, son el incremento de las dendritas en la región CA1 del hipocampo. También se ha reportado disminución del declive cognitivo al iniciar la terapia estrogénica inmediatamente después de la menopausia, pero no en aquellas que se inició en forma tardía. Además se ha reportado una reducción significativa de riesgo de enfermedad de Alzheimer en mujeres postmenopáusicas en quienes se inició el remplazo hormonal en forma temprana. Todos los estudios, en roedores, primates y humanos convergen en apoyar la idea de que los estrógenos pueden prevenir los efectos deletéreos sobre la cognición, cuando se administran en una etapa temprana después del cese de la función ovárica ${ }^{20}$. 


\section{Conclusiones}

El grupo de ratas ovariectomizadas suplementadas con estrógenos demostró tener mayor capacidad de aprendizaje en una tarea de tipo espacial comparado con el grupo de ratas ovariectomizadas sin suplementación. Por lo tanto la suplementación con estradiol a la dosis administrada, es suficiente para que se reviertan los efectos de hipoestrogenismo inducidos por la ovariectomía. Se puede sugerir que se realizaron los cambios plásticos necesarios en el hipocampo dorsal para restablecer los procesos cognitivos en las ratas suplementadas. No obstante es recomendable la realización del estudio histológico para comprobar estos cambios en el hipocampo dorsal de la rata.

\section{Bibliografía}

1 McGaugh JL, Martinez JL Jr, Messing RB, Liang KC, Jensen RA, Vasquez BI, Rigter $\mathrm{H}$. Role of neurohormones as modulators of memory storage. Adv. Biochem Psychopharmacol 1982;33:123-30.

2 McGaugh JL. Hormonal influences on memory. Annu Rev Psychol. 1983;34:297-323.

3 McEwen BS. Stress and the aging hippocampus. Front Neuroendocrinol. 1999;20(1):49-70.

4 Sudo S, Wen TC, Desaki J, Matsuda S, Tanaka J, Arai T, Maeda N, Sakanaka M. Beta-estradiol protects hippocampal CA1 neurons against trainsent forebrain ischemia in gerbil. Neurosci Res. 1997;29(4):34554.

5 Bratt AM, Kelley SP, Knowles JP, Barrett J, Davis K, Davis $M$, Mittleman G. Long term modulation of HPA axis by the hippocampus. Behavioral, biochemical and immunological endpoints in rats exposed to chronic mild stress. Psychoneuroendocrinology. 2001;26(2):121-45.

6 Guyton A, Hall J. 2001. Tratado de fisiología medica. $10^{a}$ Ed. España. 820 - 826.

7 Meltzoff AN, Kuhl PK, Movellan J, Sejnowski TJ. Foundations for a new science of learning. Science 2009;11:325(5946):1340.

8 Balderas I, Ramírez-Amaya V, Bermúdez-Rattoni F. 2004 Cambios morfológicos asociados a la memoria. Revista de Neurología; 38 (10): 944-948.

9 Sousa N, Almeida OF. Corticosteroids. Sculptors of the hippocampal formation. Rev Neurosci. 2002;13(1):59-84.

10 Barley CH, Bartsh D. Kandel E R. Toward a molecular definition of long - term memory storage. Proc Natl Acad Sci, 1996; 93. 13445 - 13452.

11 Amaral, D; Lavenex P 2006. Hippocampal Neuroanatomy. The Hippocampus Book. Oxford University Press.
12 Cutsuridis V, Wennekers T. Hippocampus, microcircuits and associative memory. Neural Netw. 2009;22(8):110-118.

13 Lee $\mathrm{E}$, Son $\mathrm{H}$. Adult hippocampal neurogénesis and related neurotrophic factors. BMB Report. 2009, 31;42(5):239-244.

14 Zhao C, Deng A, Cage FH. Mechanisms and functional implications of adult neurogenesis. Cell 2008; 132:645-660.

15 Woolley CS, Wenzel HJ, Schwartzkroin PA. Estradiol increases the frequency of multiple synapse boutons in the hippocampal CA1 region of the adult female rat. J Comp Neurol. 1996;373(1):108-117.

16 Tanapt P, Galea LA, Gould E. Stress inhibits the proliferation of granule cell precursors in the developing dentate gyrus. Int J Dev Neurosci. 1998;16(3-4): 235-239.

17 Woolley CS. Acute effects of estrógeno on neuronal physiology. Annu Rev Pharmacol Toxicol. 2007; 47:657-680.

18 Birzniece V, Backstrom T, Johansson IM, Lindblad $C$, Lundgren $P$, Lofgren $M$, Olsson $T$, Ragagnin $G$, Taube $M$, Turkmen $S$, Wahlstrom $G$, Wang MD, Wihlback AC, Zhu D. Neuroactive steroid effects on cognitive functions with a focus on the serotonin and GABA systems. Brain Research Reviews.2006; 51: 212-239.

19 Scharfman HE, MacLusky NJ. Estrogen and brain derived neurotrophic factor (BDNF) in hippocampus complexity of steroid-hormone growth factor interactions in the adult CNS. Front Neuroendocrinol. 2006;27(41):415-435.

20 Shumaker SA, Legautl C, Rapp SR. 2003. Estrogen plus progestin and the incidence of dementia and mild cognitive impairment in postmenopausal women. The Women's Health Initiative memory study: a randomized clinical trial. ACC Current Journal Review. 2003:12:30-31 